\title{
Long-range scans and many-beam effects for high-resolution $x$-ray diffraction from multilayered structures: Experiment and theory
}

\author{
T. A. Alexeeva,,${ }^{1,2}$ A. I. Benediktovich, ${ }^{1}$ I. D. Feranchuk, ${ }^{1}$ T. Baumbach, ${ }^{2}$ and A. Ulyanenkov ${ }^{3}$ \\ ${ }^{1}$ Department of Theoretical Physics, Belarusian State University, 4 Fr. Skariny av., 220030 Minsk, Republic of Belarus \\ ${ }^{2}$ University Karlsruhe, Engesserstrasse 15, 76131 Karlsruhe, Germany \\ ${ }^{3}$ Bruker AXS GmbH, Östliche Rheinbrückenstrasse 49, 76187 Karlsruhe, Germany \\ (Received 13 December 2007; revised manuscript received 13 March 2008; published 22 May 2008)
}

\begin{abstract}
A covariant matrix method based on many-beam dynamical x-ray diffraction is reported for simulation of the wide x-ray diffraction profiles from multilayered crystalline samples both in coplanar and noncoplanar geometries. Because of many-wave scattering, the approximations of the second-order on x-ray polarizability both for dispersion equations and boundary conditions are taken into account. The influence of various factors on calculation of the x-ray profiles containing multiple Bragg reflections is investigated.
\end{abstract}

DOI: 10.1103/PhysRevB.77.174114

PACS number(s): 61.05.cc, 61.05.cp, 68.65.Ac

\section{INTRODUCTION}

Thin crystalline films and multilayers are widely used in modern nanoscale manufacturing throughout the semiconductor, coating, optical, and infrared industries. The broad use of these structures requires adequate methods for characterization and quality control as well as for development of electronic and optical devices with predictable properties. These requirements have led to advances in application of such characterization techniques ${ }^{1}$ as photoluminescence (PL), reflection high energy electron diffraction, transmission electron microscopy, ${ }^{2}$ scanning tunneling microscopy, extended $\mathrm{x}$-ray absorption fine structure, and others. Highresolution x-ray diffraction ${ }^{3}$ (HRXRD) occupies a special place among these methods due to its nondestructive character, suitability of the $\mathrm{x}$-ray wavelength for investigating nanometer and subnanometer length scales, and specific features of the diffraction process on the perfect atomic arrangement in the studied samples. This technique incorporates the fitting of experimental HRXRD profiles by theory taking into account the interference of scattered $\mathrm{x}$-ray waves on the deformations of crystallographic lattice and scattering of $x$ rays at internal interfaces to obtain the parameters of the studied samples.

The essential advantage of the HRXRD method is that the dynamical range of $\mathrm{x}$-ray measurements is very high in modern instruments and synchrotron sources, which makes it possible to measure the diffraction peaks up to the relative precision $\xi_{\text {exp }} \sim 10^{-7}$, and thus the theoretical calculations have to be as precise as $\xi_{t h} \sim \xi_{\text {exp }}$ to accurately evaluate sample parameters. This measurement precision and high perfection of the investigated structures result in detailed experimental curves, containing plenty of information and reflecting the complex scattering of $\mathrm{x}$ rays within the sample. This fact, in turn, demands a precise theoretical interpretation of the measured data taking into account numerous dynamical effects for the $\mathrm{x}$ rays inside the sample.

Growing attention has also been recently paid to the measurements and interpretation of long-range scans (LRS) in the high-resolution diffraction setup. These measurements are carried out in a wide angular range of incidence or exit beams, which covers a large number of Bragg reflections
$(>3)$. The analysis of these profiles allows the very accurate interpretation of the distortions in crystallographic lattice (see, for example, Refs. 4-7, and citations therein). The LRS measurements play an essential role in the so-called x-ray surface crystallography, ${ }^{8}$ where multiple crystal truncation rods (CTRs) are measured and evaluated. In contrast to CTRs, which scan reciprocal space with conservation of the lateral component of the wave vector, the LRSs may also pass through the reciprocal lattice points belonging to different truncation rods. Both CTRs and LRSs require a precise data interpretation in the vicinity of the Bragg peaks (conventional dynamical diffraction theory is applicable) and in the intermediate regions between the peaks (kinematical approach is applicable).

The interpretation of LRS is a typical subject for phase analysis in powder diffractometry, where $\mathrm{x}$ rays are scattered from a sample's monocrystalline blocks of small size. In this case, the position and the intensity of the Bragg peaks from a crystallographic lattice are calculated on the basis of kinematical diffraction theory. In the HRXRD setup, however, the theoretical analysis of LRS profiles from multilayers faces some specific problems. First, the dimension of monocrystalline layers is large enough (up to centimeters), and dynamical diffraction theory (DDT) has to be used for a description of the Bragg peak's amplitude, width, and position. ${ }^{9}$ Second, using a conventional two-wave approximation of DDT, the calculations are strongly related to one definite Bragg reflection, which makes a consistent analysis of the entire LRS containing several Bragg peaks impossible.

The systematic study on LRS with the use of a dynamical approach was started two decades ago. ${ }^{10-14}$ Recently, this problem has been considered in several works ${ }^{4-7,15}$ for deriving an effective algorithm for simulation of LRS, which can be applicable for modern HRXRD methods. Within the accuracy provided by the first order of $x$-ray polarizability, the problem has been resolved in Ref. 7. In this work, the twobeam DDT is utilized for simulation of LRS near the Bragg peaks, and the kinematical theory is used in intermediate angular regions. ${ }^{16}$ The effectiveness of this approach has been recently reported in Ref. 17 by comparison with experimental results. Another approach for LRS has been developed in Refs. 5 and 6 by means of the generalization and 
numerical solution of the Takagi-Taupin equations, which do not take the specular waves into consideration. A novel method for the calculation of LRS has recently been proposed in Ref. 18. To take into account many-wave effects using the DDT approach, the sample is divided into a sequence of very thin slabs parallel to the surface and the diffracted intensity is calculated by matrix formalism. In this approach, a required accuracy is achieved for a large number of the artificial slabs, which essentially increases the volume of simulations.

Let us summarize the principal approximations used in HRXRD interpretation methods. The most widely used approach is a conventional DDT based on the two-wave approximation and on the replacement of the fourth-order dispersion surface by a quadratic equation. ${ }^{9}$ Physically, it neglects the specularly reflected waves from internal interfaces. This approximation is also used in Takagi-Taupin equations ${ }^{19}$ for $\mathrm{x}$-ray diffraction from distorted crystals. The accuracy of the conventional DDT is determined by x-ray polarizability of sample material $\xi_{t h} \sim|\chi| \sim 10^{-5}$. In the conventional DDT approach, two variables are used to perform simulation: the incidence angle of $\mathrm{x}$ rays $\omega_{i}$ and deviation parameter $\alpha_{h}$ from the exact Bragg condition for a selected reciprocal lattice vector. This is not satisfactory for a covariant description of LRS, which contains several Bragg peaks, even after the introduction of a special correction ${ }^{20}$ for the deviation parameter. ${ }^{4}$ The approach is also not applicable for grazing-incidence noncoplanar x-ray diffraction or HRXRD in the region far from the Bragg peaks.

The consistent theory of HRXRD in distorted crystals and multilayers within two-beams approximation is based on various modifications of the matrix method for the calculation of x-ray wave fields (see Ref. 21, and citations therein). In this method, contrary to conventional DDT, all of four roots of the dispersion equation are used, which permits the interpretation of diffraction profiles with an accuracy up to $\sim|\chi| \sim 10^{-4}-10^{-5}$ for an arbitrary geometry but within the angular scanning range limited to the neighborhood of the Bragg peaks. The matrix methods, which are used for the calculation of $\mathrm{x}$-ray scattering from superlattices with a large number of period repetitions and in grazing-incidence geometries, cause some numerical problems, which can be overcome by the proposed recursive matrix method. ${ }^{21}$ The volume of the calculations in the matrix method does not essentially differ from the one in the conventional DDT technique.

Despite the good results for local scans near a Bragg peak, the two-beam approximation cannot be directly used for the covariant description of LRS. According to the analysis done in Ref. 4, many-beam diffraction (MBD) has to be taken into account in this case along with the accounting of the second-order corrections $\xi_{2} \sim|\chi|^{2}$ for the solution of the Maxwell equations. This assumes the consideration of the longitudinal components of $\mathrm{x}$-ray wave fields inside the sample as well as more precise boundary conditions at interfaces. It was mentioned in Ref. 14, that the effect of these corrections can be essential in the regions between the Bragg peaks, where the x-ray intensity is of order $\xi_{1} \sim|\chi|$ with respect to the intensity on the peak.

In the present work, the many-beam dynamical diffraction theory is proposed for covariant (i.e., uniformly applicable for the entire angular scanning region and for an arbitrary polarization of the wave fields) description of LRS, which also takes into account all the required corrections to provide an accuracy of the calculated diffraction profiles up to $\xi_{t h}$ $\sim|\chi|^{2}$. The aim of the investigation is to develop a MBD method suitable for the applications and convenient for the experimental LRS data fitting. We have found an analytical approximation for the dispersion equation in the MBD case, which is valid for arbitrary wave vectors and crystal parameters. As a result, the LRS profile is defined solely by a single variable $\omega_{i}$ within the entire angular range, and is calculated uniformly both near the Bragg peaks and between them. The covariant matrix method (CCM) is extended for the MBD case with corrections up to the order of $\sim|\chi|^{2}$ and for the boundary conditions applicable for all measurement geometries, except for the special case when multiple Bragg diffraction occurs (Bragg conditions coincide for three or more crystallographic planes). In Sec. II, the analytical solution for the dispersion equation in the MBD case is obtained, which provides the required accuracy for the equation roots within the entire region of the sample parameters variation. The boundary conditions for all waves taking part in the scattering process are resolved in Sec. III. In Sec. IV, several numerical examples demonstrating the advantages of the proposed technique are presented and the accuracy of the method is proved by comparison with experimental LRS, containing 16 Bragg reflections from a $22 \mathrm{~nm}$ layer of $\mathrm{YBa}_{2} \mathrm{Cu}_{3} \mathrm{O}_{7}$ on a $\mathrm{SrTiO}_{3}$ substrate.

\section{APPROXIMATE ANALYTICAL SOLUTION FOR MBD DISPERSION EQUATION}

We start with the expressions describing the X-ray wave fields $\vec{E}_{\vec{K}}(\vec{r}, \omega)$ and $\vec{D}_{\vec{K}}(\vec{r}, \omega)$ for monochromatic radiation of frequency $\omega$ in a nonmagnetic ideal crystal as follows:

$$
\vec{E}_{\vec{K}}(\vec{r}, \omega)=\sum_{\vec{H}} \vec{E}_{(\vec{K}+\vec{H})} e^{i(\vec{K}+\vec{H}) \cdot \vec{r}}, \quad \vec{D}_{\vec{K}}(\vec{r}, \omega)=\sum_{\vec{H}} \vec{D}_{(\vec{K}+\vec{H})} e^{i(\vec{K}+\vec{H}) \cdot \vec{r}},
$$

where the summation is performed over the infinite set of the vectors $\vec{H}=0 ; \vec{h} ; \vec{g} \ldots$ of the crystal reciprocal lattice.

This form of the wave fields is defined by the Bloch theorem for a three-dimensional (3D) periodical crystal. The coefficients in Eq. (1) satisfy the infinite system of algebraic equations, which follows from the Maxwell equations with periodic $\mathrm{x}$-ray polarizability of the crystal as follows:

$$
\begin{gathered}
\chi(\vec{r}, \omega)=\sum_{\vec{H}} \chi_{\vec{H}} e^{i \vec{H} \cdot \vec{r}}, \\
K^{2} \vec{E}_{\vec{K}}-\vec{K}\left(\vec{K} \cdot \vec{E}_{\vec{K}}\right)-k_{0}^{2} \vec{D}_{\vec{K}}=0, \quad(\vec{K}+\vec{H}) \cdot \vec{D}_{(\vec{K}+\vec{H})}=0, \\
\vec{D}_{\vec{K}}=\vec{E}_{\vec{K}}+\sum_{\vec{H}} \chi_{\vec{H}} \vec{E}_{(\vec{K}-\vec{H})} .
\end{gathered}
$$

The wave vector $\vec{K}$ is assumed to belong to the Brillouin zone of reciprocal space, corresponding to the wave vector of 
the incident $\mathrm{x}$-ray wave with wavelength $\lambda$ as follows:

$$
\vec{K} \approx \vec{k} \equiv k_{0} \vec{n}, \quad k_{0}=\omega / c=\frac{2 \pi}{\lambda} .
$$

The Fourier components of the electric field in Eq. (3) can be excluded without essential loss of accuracy by representing the constitutive equation as:

$$
\vec{E}_{K}=\vec{D}_{\vec{K}}-\sum_{\vec{H}} \chi_{\vec{H}} \vec{E}_{(\vec{K}-\vec{H})},
$$

and performing two sequential iterations as follows:

$$
\vec{E}_{\bar{K}} \approx \vec{D}_{\bar{K}}-\sum_{\vec{H}} \chi_{\vec{H}} \vec{D}_{(\vec{K}-\vec{H})}+\sum_{\vec{H}} \sum_{\vec{G}} \chi_{\vec{H}} \chi_{\vec{G}} \vec{D}_{(\vec{K}-\vec{H}-\vec{G})} .
$$

Substituting Eq. (5) in Eq. (3) and using the transverse condition for the vectors $\vec{D}$, we obtain the expressions

$$
\begin{gathered}
\left(K^{2}-k_{0}^{2}\right) \vec{D}_{\vec{K}}-K^{2} \sum_{\vec{H}} \widetilde{\chi}_{\vec{H}} \vec{D}_{(\vec{K}-\vec{H})}+\vec{K} \sum_{\vec{H}} \tilde{\chi}_{\vec{H}}\left(\vec{K} \cdot \vec{D}_{(\vec{K}-\vec{H})}\right)=0, \\
\widetilde{\chi}_{\vec{H}} \equiv \chi_{\vec{H}}-\sum_{\vec{G}} \chi_{\vec{G}} \chi_{(\vec{H}-\vec{G})},
\end{gathered}
$$

which are different from commonly used equations for DDT due to the additional terms related to both the change of the wave field polarization in the crystal and to the renormalization of Fourier components of x-ray polarizability $\widetilde{\chi}_{H}$. This renormalization is negligible in most cases but it can be essential, for example, for so-called quasiforbidden Bragg reflections where it results in a weak but nonzero intensity of the diffracted waves.

Equation (6) are the infinite set of vector equations. The presence of small parameters in these equations (the components of x-ray polarizability $\widetilde{\chi}_{H}$ ) permits the construction of the iteration method for the solution of the system, where the dispersion equation for the two-beam diffraction is taken as an initial approximation. To build the iteration scheme, the definition of the wave field polarization has to be modified. The issue is that the expression (6) for the two-beam case lead to the dispersion equation of eighth order. ${ }^{9}$ The problem is then reduced to two independent equations of fourth order, if the wave field polarizations are supposed to be connected to the particular reciprocal lattice vector $\vec{H}$. However, for simulation of LRS containing several Bragg reflections, the polarizations have to be defined in coordinates which are not related to some crystallographic planes of the crystal. Below, the analytical solution for the dispersion equation for twobeam diffraction is obtained in covariant form, which is not connected to any reciprocal lattice vector.

Let us consider Eq. (6) with two amplitudes only: direct $\vec{D}_{\vec{K}}$ and diffracted $\vec{D}_{\vec{K}_{h}^{-}}=\vec{D}_{(\vec{K}+\vec{h})}$ waves, corresponding to the certain reciprocal lattice vector $\vec{h}$ as follows:

$$
\left\{K^{2}\left(1-\widetilde{\chi}_{0}\right)-k_{0}^{2}\right\} \vec{D}_{\vec{K}}-\widetilde{\chi}_{-}\left[K^{2} \vec{D}_{K_{h}}-\vec{K}\left(\vec{K} \cdot \vec{D}_{\vec{K}_{h}}^{\vec{h}}\right)\right]=0,
$$

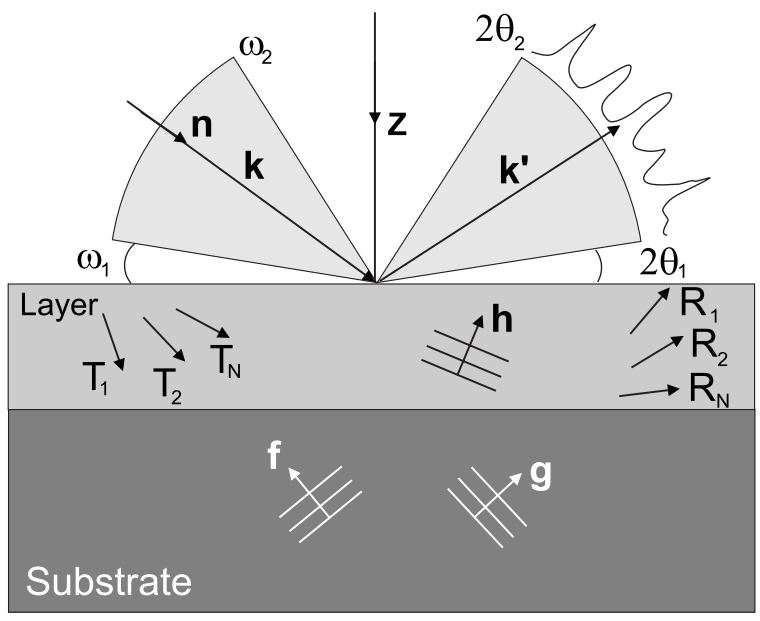

FIG. 1. Schematic view of the x-ray Bragg many-beam diffraction from a thin layer on the substrate.

$$
\begin{aligned}
& \left\{K_{\vec{h}}^{2}\left(1-\widetilde{\chi}_{0}\right)-k_{0}^{2}\right\} \vec{D}_{\hat{K}_{h}}^{\vec{h}}-\widetilde{\chi}_{\hat{h}}\left[K_{\vec{h}}^{2} \vec{D}_{\vec{K}}-\vec{K}_{\vec{h}}^{\vec{h}}\left(\vec{K}_{\vec{h}} \cdot \vec{D}_{\vec{K}}\right)\right]=0, \\
& \vec{K}_{\vec{h}}=\vec{K}+\vec{h} .
\end{aligned}
$$

We do not use in these equations the conventional substitution $\left(1-\chi_{0}\right)^{-1} \approx\left(1+\chi_{0}\right)$, which does not satisfy the desired in this work accuracy. ${ }^{4}$ Usually, the coordinate system of the diffraction plane, defined by the vectors $\vec{K}$ and $\vec{K}_{h}$, is used to resolve the problem. Then two polarization eigenstates of the direct and the diffracted waves ( $\sigma$ and $\pi$ polarizations) are distinguished, which follow from the algebraic system of scalar equations. However, for a wide angular range of LRS, the vector $\vec{K}$ may occur in the reciprocal lattice points, which correspond to the reciprocal lattice vectors $\vec{g} \neq \vec{h}$ and thus other polarizations are defined (the case of noncoplanar diffraction). Therefore, the covariant representation for the wave field polarizations has to be obtained in Eq. (8), which is not related to the diffraction plane of the certain vector $\vec{h}$. We have to note that the covariant approach has also been used for x-ray polarizations in arbitrary measurement geometry in the case of two-beam diffraction. ${ }^{22}$

Using the second equation in Eqs. (8), the amplitude of the diffracted wave can be defined and then the vector equation for the amplitude of the direct wave is

$$
\begin{gathered}
\left\{K^{2}\left(1-\tilde{\chi}_{0}\right)-k_{0}^{2}\right\}\left\{K_{\vec{h}}^{2}\left(1-\tilde{\chi}_{0}\right)-k_{0}^{2}\right\} \vec{D}_{\vec{K}}-\tilde{\chi}_{\vec{h}} \tilde{\chi}_{-} \vec{h}\left\{K^{2} K_{\vec{h}}^{2} \vec{D}_{\vec{K}}\right. \\
\left.-\left(\vec{K}_{\vec{h}} \cdot \vec{D}_{\vec{K}}\right)\left[\vec{K}_{\vec{h}} K^{2}-\vec{K}\left(\vec{K} \cdot \vec{K}_{\vec{h}}\right)\right]\right\}=0 .
\end{gathered}
$$

We assume in Eq. (9),

$$
\vec{K}=K_{0} \vec{n}, \quad \vec{K}_{\vec{h}} \approx K_{h} \vec{n}_{h}, \quad \vec{n}_{\vec{h}}=\frac{K_{0}}{K_{h}} \vec{n}+\frac{\vec{h}}{K_{h}} .
$$

In the x-ray diffraction experiments, the scattering geometry is described by two vectors: the unit vector $\vec{n}$ and the vector $\vec{Z}$ of the inward normal to the sample surface or interface (Fig. 1). These vectors are not related to the certain Bragg plane and can be used for a description of the polarization 
states of the direct wave corresponding to the wave vector $\vec{K}$ as follows:

$$
\begin{gathered}
\vec{D}_{K}=D_{1} \vec{e}_{1}+D_{2} \vec{e}_{2}, \quad \vec{e}_{1}=\frac{[\vec{n} \times \vec{Z}]}{\|[\vec{n} \times \vec{Z}]\|}, \\
\vec{e}_{2}=\left[\vec{e}_{1} \times \vec{n}\right]=\vec{Z}-\vec{n}(\vec{n} \cdot \vec{Z}) .
\end{gathered}
$$

Then the equations for scalar amplitudes are expressed as

$$
\begin{aligned}
& \left\{\frac{K^{2}}{k_{0}^{2}}\left(1-\tilde{\chi}_{0}\right)-1\right\}\left\{\frac{K_{\vec{h}}^{2}}{k_{0}^{2}}\left(1-\tilde{\chi}_{0}\right)-1\right\} D_{1} \\
& -\frac{K_{h}^{2} K_{0}^{2}}{k_{0}^{4}} \tilde{\chi}_{h} \tilde{\chi}_{-h} \vec{h}\left[1-\left(\vec{n}_{\vec{h}} \cdot \vec{e}_{1}\right)^{2}\right] D_{1} \\
& \left.-\left(\vec{n}_{h} \cdot \vec{e}_{1}\right)\left(\vec{n}_{\vec{h}} \cdot \vec{e}_{2}\right) D_{2}\right\}=0 \text {, } \\
& \left\{\frac{K^{2}}{k_{0}^{2}}\left(1-\tilde{\chi}_{0}\right)-1\right\}\left\{\frac{K_{\vec{h}}^{2}}{k_{0}^{2}}\left(1-\tilde{\chi}_{0}\right)-1\right\} D_{2} \\
& -\frac{K_{h}^{2} K_{0}^{2}}{k_{0}^{4}} \tilde{\chi}_{\vec{h}} \tilde{\chi}-\vec{h}\left\{\left[1-\left(\vec{n}_{\vec{h}} \cdot \vec{e}_{2}\right)^{2}\right] D_{2}\right. \\
& \left.-\left(\vec{n}_{\vec{h}} \cdot \vec{e}_{1}\right)\left(\vec{n}_{\vec{h}} \cdot \vec{e}_{2}\right) D_{1}\right\}=0 .
\end{aligned}
$$

Evidently, the wave vectors corresponding to the wave polarization eigenstates in the crystal do not depend on the choice of the basic polarization vectors, and the determinant of Eq. (11) results in recognized dispersion equations for $\sigma$ and $\pi$ states of polarization as follows:

$$
\begin{gathered}
{\left[\frac{K^{2}}{k_{0}^{2}}\left(1-\tilde{\chi}_{0}\right)-1\right]\left[\frac{K_{\vec{h}}^{2}}{k_{0}^{2}}\left(1-\tilde{\chi}_{0}\right)-1\right]-\frac{K_{h}^{2} K_{0}^{2}}{k_{0}^{4}} \widetilde{\chi}_{h} \tilde{\chi}-\hat{h}\left[C_{s}^{h}\right]^{2}=0,} \\
s=\sigma, \pi, \quad C_{\sigma}^{h}=1, \quad C_{\pi}^{h}=\cos 2 \theta_{B}^{h}=\left(\vec{n} \cdot \vec{n}_{h}\right) .
\end{gathered}
$$

However, the explicit form of the amplitudes from Eq. (9) depends on the choice of the basis and the amplitudes are defined as

$$
\begin{gathered}
\vec{D}_{\vec{K}}^{\sigma}=D_{\vec{K}}^{\sigma}\left[\vec{e}_{1} \cos \delta_{h}-\vec{e}_{2} \sin \delta_{h}\right], \\
\vec{D}_{\vec{K}}^{\pi}=D_{\vec{K}}^{\pi}\left[\vec{e}_{1} \sin \delta_{h}+\vec{e}_{2} \cos \delta_{h}\right], \\
\vec{D}_{\vec{K}_{h}^{*}}^{s}=\left[K_{\vec{h}}^{2} / k_{0}^{2}\left(1-\tilde{\chi}_{0}\right)-1\right]^{-1} \widetilde{\chi}_{h}\left[\vec{D}_{\vec{K}}^{s}-\vec{n}_{h}\left(\vec{n}_{\vec{h}} \cdot \vec{D}_{\vec{K}}^{s}\right)\right] \frac{K_{h}^{2}}{k_{0}^{2}},
\end{gathered}
$$

with the scalar amplitudes $D_{\vec{K}}^{s}$ and noncoplanar angle $\delta_{h}$ between the wave vector of the diffracted wave and the incidence plane defined by the vectors $\vec{n}, \vec{Z}: \cos \delta_{h}=([\vec{n} \times \vec{Z}] \cdot[\vec{n}$ $\left.\times \vec{n}_{h}\right]$ ).

The most convenient choice for the wave vector in the crystal $^{21}$ assumes the projection of $\vec{K}_{\perp}$ onto the plane perpendicular to the surface normal $\vec{Z}$ to be independent, i.e., the component $\vec{K}_{\perp}$ remains continuous at interfaces. Thus, Eq.
(12) leads to the solution for normal projection of wave vector $u=K_{z} / k_{0}$. We use here the results from Ref. 21, where Eq. (12) is written in the following form:

$$
\left[u^{2}-\gamma_{0}^{2}-\tilde{\chi}_{0}\right]\left[\left(u+\psi_{h}\right)^{2}-\gamma_{h}^{2}-\tilde{\chi}_{0}\right]-\frac{K_{h}^{2} K_{0}^{2}}{k_{0}^{4}} \tilde{\chi}_{h} \tilde{\chi}_{-h} C_{s}^{2}=0,
$$

and diffraction parameters are defined as

$$
\begin{gathered}
\gamma_{0}^{2}=\frac{k_{0}^{2}-K_{\perp}^{2}}{k_{0}^{2}}, \quad \gamma_{h}^{2}=\frac{k_{0}^{2}-(\vec{K}+\vec{h})_{\perp}^{2}}{k_{0}^{2}}, \quad \psi_{h}=\frac{(\vec{h} \cdot \vec{Z})}{k_{0}}, \\
\alpha_{h}=\frac{(\vec{K}+\vec{h})^{2}-K^{2}}{k_{0}^{2}}=\left(\gamma_{0}+\psi_{h}\right)^{2}-\gamma_{h}^{2} .
\end{gathered}
$$

The parameter $\alpha_{h}$ describes the deviation of the wave vector from the exact Bragg condition for vector $\vec{h}$, and the redefinition $\widetilde{\chi}_{0} \rightarrow \widetilde{\chi}_{0}+\widetilde{\chi}_{0}^{2}$ in Eq. (12) is used. However, for the calculation of LRS, the parameter $\gamma_{h}$ has to be calculated from Eq. (15) without using $\alpha_{h}$ unlike the usual procedure in the HRXRD case. ${ }^{21}$

To solve Maxwell equations with required precision $\sim\left|\chi_{h}\right|^{2}$, all four roots of the dispersion equation (14) have to be considered, and the analytical Cartan formulas or the numerical solution of the fourth-order equation are used to obtain the results. In most cases of LRS, the angle regions $\gamma_{0}$ $\sim \gamma_{h} \sim \sqrt{\left|\tilde{\chi}_{0}\right|}$ (grazing-incidence diffraction) are less important, and the approximate analytical solutions for the roots are used. These solutions are obtained by iterations with a small parameter

$$
Q_{s}^{h} \equiv \frac{K_{h}^{2} K_{0}^{2}}{k_{0}^{4}} \widetilde{\chi}_{h} \widetilde{\chi}_{-h}\left[C_{s}^{h}\right]^{2},
$$

and using the initial approximation taken from the two-beam method. Then the analytical expressions are derived with a desired accuracy for the roots of Eq. (14), which are located closely to the dispersion surface of vector $\vec{K}$ as follows:

$$
u_{1,2 s}^{h} \approx \pm u_{0}+x_{1,2 s}^{h}, \quad u_{0}=\sqrt{\gamma_{0}^{2}+\tilde{\chi}_{0}} .
$$

Here the values $x_{1,2 s}^{h}, u_{0}$ coincide with the standard solutions for two-beam diffraction, ${ }^{21}$ corresponding to the deviation parameters $\alpha_{h}^{ \pm}=\left(u_{0} \pm \psi_{h}\right)^{2}-\gamma_{h}^{2}-\tilde{\chi}_{0}$. Similarly, the roots, associated with the dispersion surface for $\vec{K}+\vec{h}$, are

$$
u_{3,4 s}^{h} \approx-\psi_{h} \pm u_{h}+x_{3,4 s}^{h}, \quad u_{h}=\sqrt{\gamma_{h}^{2}+\tilde{\chi}_{0}},
$$

with the deviation parameters $\widetilde{\alpha}_{h}=\left(u_{h} \pm \psi_{h}\right)^{2}-u_{0}^{2}$.

Now we derive the analytical expressions for the wave field amplitudes in the crystal, assuming a connection of waves scattered from different Bragg planes. The number of waves $N$ to be considered for LRS calculations is defined by the number of Bragg peaks located in the simulated angular interval. Let us consider the MBD equations with amplitudes corresponding to the reciprocal lattice vectors $\vec{h}, \vec{g}, \ldots$ as follows: 


$$
\begin{aligned}
& U \vec{D}_{\vec{K}}-\widetilde{\chi}_{-}\left[K^{2} \vec{D}_{\hat{K}_{h}}-\vec{K}\left(\vec{K} \cdot \vec{D}_{\hat{K}_{h}}^{\vec{h}}\right)\right] \\
& -\widetilde{\chi}_{-\vec{g}}\left[K^{2} \vec{D}_{K_{\vec{g}}}-\vec{K}\left(\vec{K} \cdot \vec{D}_{K_{\vec{g}}}\right)\right]-\cdots=0, \\
& U_{\vec{h}} \vec{D}_{\hat{K}_{\vec{h}}}-\widetilde{\chi}_{\vec{h}}\left[K_{\vec{h}}^{2} \vec{D}_{\vec{K}}-\vec{K}_{\vec{h}}\left(\vec{K}_{\vec{h}} \cdot \vec{D}_{\vec{K}}\right)\right] \\
& -\widetilde{\chi}_{\vec{h}-\vec{g}}\left[K_{\vec{h}}^{2} \vec{D}_{\vec{K}_{\vec{g}}}-\vec{K}_{\vec{h}}\left(\vec{K}_{\vec{h}} \cdot \vec{D}_{\vec{K}_{\vec{g}}}\right)\right]-\cdots=0, \\
& U_{\vec{g}} \vec{D}_{K_{\vec{g}}}-\tilde{\chi}_{\vec{g}}\left[K_{\vec{g}}^{2} \vec{D}_{K}-\vec{K}_{\vec{g}}\left(\vec{K}_{\vec{g}} \cdot \vec{D}_{K}\right)\right] \\
& -\widetilde{\chi}_{\vec{g}-h}\left[K_{\vec{g}}^{2} \vec{D}_{\vec{K}_{\vec{h}}}-\vec{K}_{\vec{g}}\left(\vec{K}_{\vec{g}} \cdot \vec{D}_{\vec{K}_{\vec{h}}}\right)\right]-\cdots=0,
\end{aligned}
$$

with $U=K^{2}-k_{0}^{2}\left(1+\tilde{\chi}_{0}\right) ; \quad U_{\vec{h}}=K_{\vec{h}}^{2}-k_{0}^{2}\left(1+\widetilde{\chi}_{0}\right) ; \ldots$

As mentioned above, the effective many-wave interaction is omitted from consideration, i.e., the Bragg condition is fulfilled simultaneously for two wave vectors only: $K^{2} \approx K_{\vec{h}}^{2}$ $\neq K_{\vec{g}}^{2}$ or $K^{2} \approx K_{\vec{g}}^{2} \neq K_{\vec{h}}^{2}$. We demonstrate below that diffracted wave fields are defined by the amplitudes $\vec{D}_{K}$, and the interaction between them due to polarizability $\tilde{\chi}_{\vec{g}-\vec{h}}$ can be neglected. First, we consider a three-wave case with coplanar vectors $\vec{h}, \vec{g}$, for which the determinant of Eq. (19) for $\sigma$ polarization is the following:

$$
\begin{gathered}
U\left(1-\frac{\left|\tilde{\chi}_{\vec{g}-\vec{h}}\right|^{2} K_{\vec{h}}^{2} K_{\vec{g}}^{2}}{U_{\vec{h}}^{2} U_{\vec{g}}}\right)-\frac{\left|\tilde{\chi}_{h}^{\vec{h}}\right|^{2} K^{2} K_{\vec{h}}^{2}}{U_{\vec{h}}}-\frac{\left|\tilde{\chi}_{\vec{g}}\right|^{2} K^{2} K_{\vec{g}}^{2}}{U_{\vec{g}}} \\
+\frac{K^{2} K_{\vec{h}}^{2} K_{\vec{g}}^{2}}{U_{\vec{h}}^{*} U_{\vec{g}}}\left[\widetilde{\chi}_{\vec{g}} \tilde{\chi}-\vec{h} \widetilde{\chi}_{\vec{h}-\vec{g}}+\tilde{\chi}_{-\vec{g}} \tilde{\chi}_{\vec{h}} \tilde{\chi}_{\vec{g}-\vec{h}}\right]=0 .
\end{gathered}
$$

As follows from Eq. (20), the consideration of the terms $\sim \widetilde{\chi}_{\vec{g}-\vec{h}}$ near the Bragg peak (e.g., $\left.U_{\vec{g}} \gg U \sim U_{\vec{h}} \sim K^{2}\left|\chi_{0}\right|\right)$ leads to the shift of the angular position of the peak by the value $\sim\left|\chi_{0}\right|^{2}$, which is beyond the precision of the HRXRD technique. In between the peaks $U_{\vec{g}} \sim U_{\vec{h}} \gg U \sim K^{2}\left|\chi_{0}\right|$, these terms are $\sim\left|\chi_{0}\right|^{3}$, which is beyond the dynamical range of the HRXRD. The similar yet more cumbersome analysis may be provided for an arbitrary number of the waves involved in the scattering process and for arbitrary polarization.

Thus, neglecting the interaction of the diffracted waves, the $N$-equations system is reduced to the single equation for $\overrightarrow{D_{K}}$ as follows:

$$
\begin{aligned}
U \vec{D}_{\bar{K}} & -\frac{\tilde{\chi}_{\hat{h}} \tilde{\chi}_{-h}}{U_{\vec{h}}}\left\{K^{2} K_{\vec{h}}^{2} \vec{D}_{\vec{K}}-\left(\vec{K}_{\vec{h}} \cdot \vec{D}_{\vec{K}}\right)\left[\vec{K}_{\vec{h}} K^{2}-\vec{K}\left(\vec{K} \cdot \vec{K}_{\vec{h}}\right)\right]\right\} \\
& \quad-\frac{\widetilde{\chi}_{\vec{g}} \widetilde{\chi}_{-\vec{g}}}{U_{\vec{g}}}\left\{K^{2} K_{\vec{g}}^{2} \vec{D}_{\vec{K}}-\left(\vec{K}_{\vec{g}} \cdot \vec{D}_{\vec{K}}\right)\left[\vec{K}_{\vec{g}} K^{2}-\vec{K}\left(\vec{K} \cdot \vec{K}_{\vec{g}}\right)\right]\right\}-\cdots \\
= & 0 .
\end{aligned}
$$

By expanding the vector $\vec{D}_{K}$ into the series over the polarizations (10), we obtain two equations for the scalar amplitudes with the following determinant:

$$
\begin{aligned}
& \left\{\nu+A_{h}\left(\vec{n}_{\vec{h}} \cdot \vec{e}_{1}\right)^{2}+A_{\vec{g}}\left(\vec{n}_{\vec{g}} \cdot \vec{e}_{1}\right)^{2}+\cdots\right\}\left\{\nu+A_{h}\left(\vec{n}_{\vec{h}} \cdot \vec{e}_{2}\right)^{2}\right. \\
& \left.+A_{\vec{g}}\left(\vec{n}_{\vec{g}} \cdot \vec{e}_{2}\right)^{2}+\cdots\right\}-\left\{A_{\vec{h}}\left(\vec{n}_{\vec{h}} \cdot \vec{e}_{1}\right)\left(\vec{n}_{\vec{h}} \cdot \vec{e}_{2}\right)\right. \\
& \left.+A_{\vec{g}}\left(\vec{n}_{h} \cdot \vec{e}_{1}\right)\left(\vec{n}_{h} \cdot \vec{e}_{2}\right)+\cdots\right\}^{2}=0, \\
& \nu=\left\{K^{2} / k_{0}^{2}-\left(1+\tilde{\chi}_{0}\right)\right\}-A_{\vec{h}}-A_{\vec{g}}-\cdots, \\
& A_{\vec{h}}=\frac{K_{h}^{2} K_{0}^{2}}{k_{0}^{4}} \frac{\tilde{\chi}_{\vec{h}} \tilde{\chi}_{-h}}{\left\{K_{h}^{2} / k_{0}^{2}-\left(1+\tilde{\chi}_{0}\right)\right\}}, \\
& A_{\vec{g}}=\frac{K_{g}^{2} K_{0}^{2}}{k_{0}^{4}} \frac{\tilde{\chi}_{\vec{g}} \tilde{\chi}_{-\vec{g}}}{\left\{K_{\vec{g}}^{2} / k_{0}^{2}-\left(1+\tilde{\chi}_{0}\right)\right\}}, \ldots
\end{aligned}
$$

This expression can be further simplified by neglecting the products $A_{\vec{h}} \times A_{\vec{g}}, \ldots$ in the case, when the main dispersion surface (close to $\vec{K}$ ) is considered, and no intersections of three and more dispersion surfaces are present. Actually, within the entire range of the LRS, the main contribution to Eq. (22) is given by the term proportional to $\sim \nu^{2} \sim\left|\chi_{0}\right|^{2}$. In the vicinity of the particular Bragg peak (e.g., $\vec{h})$, the amplitude $A_{\vec{h}} \sim\left|\widetilde{\chi}_{h}\right|$, and other amplitudes $A_{\vec{g}}, \ldots \sim\left|\widetilde{\chi}_{h}\right|^{2}$. Thus, the products $A_{h} \times A_{\vec{g}} \sim\left|\tilde{\chi}_{h}\right|^{3}$ are beyond the limits of the accuracy considered here. In between the Bragg peaks, the products $A_{h} \times A_{\vec{g}} \sim\left|\widetilde{\chi}_{h}\right|^{4}$ become smaller and can be omitted. Then the solution of Eq. (22) delivers the values $\nu$ as follows:

$$
\begin{gathered}
\nu_{1}=0, \Rightarrow\left\{K^{2} / k_{0}^{2}-\left(1+\widetilde{\chi}_{0}\right)\right\}-A_{\vec{h}}-A_{\vec{g}}-\cdots=0, \\
\nu_{2}=-A_{\vec{h}}\left[\left(\vec{n}_{\vec{h}} \cdot \vec{e}_{1}\right)^{2}+\left(\vec{n}_{\vec{h}} \cdot \vec{e}_{2}\right)^{2}\right]-A_{\vec{g}}\left[\left(\vec{n}_{\vec{g}} \cdot \vec{e}_{1}\right)^{2}+\left(\vec{n}_{\vec{g}} \cdot \vec{e}_{2}\right)^{2}\right] \\
-\cdots=0, \Rightarrow \\
\left\{K^{2} / k_{0}^{2}-\left(1+\tilde{\chi}_{0}\right)\right\}-A_{\vec{h}}\left(\vec{n} \cdot \vec{n}_{h}\right)^{2}-A_{\vec{g}}\left(\vec{n} \cdot \vec{n}_{\vec{g}}\right)^{2}-\cdots=0 .
\end{gathered}
$$

Similarly to the case of a two-beam diffraction, these equations describe two eigenpolarizations $(s=\sigma, \pi)$ of the wave field in the crystal, however, these polarizations are not directly related to the particular reciprocal lattice vector. Introducing the notations from Eqs. (14)-(16), the dispersion equations for both polarizations are

$$
\begin{aligned}
& {\left[u^{2}-\gamma_{0}^{2}-\tilde{\chi}_{0}\right]-\frac{Q_{s}^{h}}{\left[\left(u+\psi_{h}\right)^{2}-\gamma_{h}^{2}-\tilde{\chi}_{0}\right]}-\frac{Q_{s}^{g}}{\left[\left(u+\psi_{g}\right)^{2}-\gamma_{g}^{2}-\tilde{\chi}_{0}\right]}} \\
& -\cdots=0 .
\end{aligned}
$$

The wave fields are expressed by the formulas, which are reduced to Eq. (13) when the wave vector $\vec{K}$ is close to one of the Bragg peaks as follows:

$$
\begin{array}{cl}
\vec{D}_{\vec{K}}^{\sigma}=D_{\vec{K}}^{\sigma} \vec{e}_{\sigma}, & \vec{D}_{\vec{K}}^{\pi}=D_{\vec{K}}^{\pi} \vec{e}_{\pi}, \\
\vec{e}_{\sigma}=\left[\vec{e}_{1} \Phi_{1}-\vec{e}_{2} \Phi_{2}\right], & \vec{e}_{\pi}=\left[\vec{e}_{1} \Phi_{2}+\vec{e}_{2} \Phi_{1}\right],
\end{array}
$$

with arbitrary scalar amplitudes $D_{\vec{K}}^{S}$ and 


$$
\begin{aligned}
& \Phi_{1} \equiv \frac{A_{\vec{h}}\left(\vec{n}_{\vec{h}} \cdot \vec{e}_{2}\right)^{2}+A_{\vec{g}}\left(\vec{n}_{\vec{g}} \cdot \vec{e}_{2}\right)^{2}+\cdots}{\left[A_{\vec{h}}^{2}\left(\vec{n}_{\vec{h}} \cdot \vec{e}_{2}\right)^{2}\left(\vec{n}_{\vec{h}} \cdot \vec{n}\right)^{2}+A_{\vec{g}}^{2}\left(\vec{n}_{\vec{g}} \cdot \vec{e}_{2}\right)^{2}\left(\vec{n}_{\vec{g}} \cdot \vec{n}\right)^{2}+\cdots\right]^{1 / 2}}, \\
& \Phi_{2} \equiv \frac{A_{\vec{h}}\left(\vec{n}_{\vec{h}} \cdot \vec{e}_{2}\right)\left(\vec{n}_{\vec{h}} \cdot \vec{e}_{1}\right)+A_{\vec{g}}\left(\vec{n}_{\vec{g}} \cdot \vec{e}_{2}\right)\left(\vec{n}_{\vec{g}} \cdot \vec{e}_{1}\right)+\cdots}{\left[A_{\vec{h}}^{2}\left(\vec{n}_{\vec{h}} \cdot \vec{e}_{2}\right)^{2}\left(\vec{n}_{\vec{h}} \cdot \vec{n}\right)^{2}+A_{\vec{g}}^{2}\left(\vec{n}_{\vec{g}} \cdot \vec{e}_{2}\right)^{2}\left(\vec{n}_{\vec{g}} \cdot \vec{n}\right)^{2}+\cdots\right]^{1 / 2}} .
\end{aligned}
$$

The amplitudes of the diffracted waves are calculated analogously to Eq. (13). The essential fact is that all the formulas contain the dependence on $\vec{K}$ and all the reciprocal lattice vectors are taking part in LRS simulation in a covariant way.

In the general case, the dispersion equation (24) has $4 N$ roots $u$, where $N$ is a number of considered waves. Assuming that not more than two roots occur simultaneously near the same point of the dispersion surface, i.e., the absence of the many-wave diffraction, the analytical expressions for the roots can be obtained with the accuracy $\sim\left|\tilde{\chi}_{0}\right|^{2}$ and for any wave vector $\vec{K}$ and geometry, including also a noncoplanar one. We use here the iteration scheme based on the condition $\left|Q_{s}\right| \sim\left|\tilde{\chi}_{0}\right|^{2}$, similarly to the case of two-beam diffraction in Eqs. (17) and (18). Rearranging Eq. (24),

$$
\begin{gathered}
X_{K}-\frac{Q_{s}^{h}}{X_{K_{h}}}-\frac{Q_{s}^{g}}{X_{K_{g}}^{\vec{g}}}-\cdots=0, \\
X_{K}=\left[u^{2}-\gamma_{0}^{2}-\widetilde{\chi}_{0}\right], \quad X_{K_{h}}\left[\left(u+\psi_{h}\right)^{2}-\gamma_{h}^{2}-\widetilde{\chi}_{0}\right], \\
X_{K_{g}}=\left[\left(u+\psi_{g}\right)^{2}-\gamma_{g}^{2}-\widetilde{\chi}_{0}\right]-\cdots,
\end{gathered}
$$

the analytical approximation for $2 N$ roots of Eq. (26) for fixed polarization and valid for any $\vec{K}$ is obtained in the following way. The asymptotic formulas for the roots are well known for the values $\vec{K}$, which are close to and far away from the Bragg peaks. In the former case, the main contribution to Eq. (26) is provided by the term, with $X_{K_{h}}, X_{K_{g}}, \ldots$ close to zero, and then the roots follow from Eqs. (17) and (18) with corresponding reciprocal lattice vector. In the latter case, the approximate value of $x$ is obtained as a sum of small $N$ terms as follows:

$$
x \approx \frac{Q_{s}^{h}}{X_{K_{h}}}+\frac{Q_{s}^{g}}{X_{K_{g}}}+\cdots .
$$

Both limiting cases may be represented in the covariant form, which delivers the expression for the roots of Eq. (26) for each polarization. For instance, four roots, which are close to the dispersion surface corresponding to the vector $\vec{K}$, are

$$
u_{1,2 s} \approx \pm u_{0}+x_{1,2 s}^{h(1)}+x_{1,2 s}^{g(1)}+\cdots,
$$

where $s=\sigma, \pi, x_{1,2 s}^{h(1)} ; x_{1,2 s}^{g(1)} ; \ldots$ follow from Eq. (17) for definite reciprocal lattice vector. The rest of $4(N-1)$ roots follow from

$$
\begin{aligned}
& u_{3,4 s}^{h} \approx-\psi_{h} \pm u_{h}+x_{3,4 s}^{h(1)}, \\
& u_{3,4 s}^{g} \approx-\psi_{g} \pm u_{g}+x_{3,4 s}^{g(1)},
\end{aligned}
$$

$\ldots$,

and parameters $x_{3,4 s}^{h(1)} ; x_{3,4 s}^{h(1)} ; \ldots$ are calculated by Eq. (18).

\section{MATRIX METHOD FOR THE BOUNDARY CONDITIONS IN MBD}

Equations (27) and (28) express analytically the monochromatic wave field in an infinite crystal for the whole range of the vector $\vec{K}$ as a superposition of the waves.

$$
\vec{D}(r)=\sum_{s=\sigma, \pi} \sum_{\nu=1}^{2 N} e^{i \vec{K}_{\perp} \cdot \vec{r}_{\perp}+i k_{0} u_{\nu, s} z}\left[\vec{e}_{\nu, s}+\sum_{j=1}^{N-1} \vec{V}_{s, \nu}^{h_{j}} e^{i \vec{h}_{j} \cdot \vec{r}}\right] D_{\nu, s} .
$$

The interaction dynamics between the x-ray wave and the crystal establishes the polarization eigenvectors $\vec{e}_{\sigma, \pi}^{\nu}$, which are not necessarily coincident with the basic polarization vectors $\vec{e}_{1,2}^{\nu}$ in the incidence plane. The vectors $\vec{V}_{s, \nu}^{h_{j}}$ are calculated by Eq. (25) with a proper root of the dispersion equation $u_{\sigma, \pi, \nu}$ as follows:

$$
\vec{V}_{s, \nu}^{h_{j}}=\frac{K_{h}^{2} K_{0}^{2}}{k_{0}^{4}} \frac{\tilde{\chi}_{h_{j}}}{\left(u_{\nu, s}+\psi_{h_{j}}\right)^{2}-\gamma_{h_{j}}^{2}-\widetilde{\chi}_{0}}\left[\vec{e}_{\nu, s}-\vec{n}_{h_{j}}\left(\vec{n}_{h_{j}} \cdot \vec{e}_{\nu, s}\right)\right],
$$

and in a similar way for other reciprocal lattice vectors.

The $4 N$ undefined amplitudes $D_{\nu, s}$ are found from the boundary conditions. In the multilayered structures, the matrix form of the boundary conditions is preferable (see Ref. 21 for the two-beam case). The undefined amplitudes $D_{\nu, s}^{n}$ for the layer $n$ are considered as the projections of the $4 N$-component vector, and each crystalline layer is characterized by x-ray polarizabilities $\chi_{0}^{n}, \chi_{H}^{n}$ (for amorphous layers $\left.\chi_{\vec{H}}^{n}=0\right)$. The reciprocal lattice vectors involved in LRS simulations differ in normal components only (normal strain),

$$
\vec{h}_{\perp}^{n}=\vec{h}_{\perp}^{n+1}, \quad \vec{g}_{\perp}^{n}=\vec{g}_{\perp}^{n+1}, \ldots, \quad h_{z}^{n} \neq h_{z}^{n+1}, \quad g_{z}^{n} \neq g_{z}^{n+1}, \ldots
$$

To calculate the wave field with an accuracy $\sim \chi_{\vec{h}}^{2}$, the general form of the boundary conditions for the normal and tangential components of the field has to be used. ${ }^{23}$ Let us consider first the electric field as follows:

$$
\begin{aligned}
{\left.\left[\left(\vec{Z} \cdot \vec{D}^{(n)}\right)-\left(\vec{Z} \cdot \vec{D}^{(n+1)}\right)\right]\right|_{z=z_{n}}=} & 0, \\
& {\left.\left[\vec{E}^{(n)}-\vec{Z}\left(\vec{Z} \cdot \vec{E}^{(n)}\right)\right]\right|_{z=z_{n}} } \\
= & {\left.\left[\vec{E}^{(n+1)}-\vec{z}\left(\vec{z} \cdot \vec{E}^{(n+1)}\right)\right]\right|_{z=z_{n}}, }
\end{aligned}
$$

where $z_{n}$ is the coordinate of the interface between layers $n$ and $(n+1) \quad\left(z_{0}=0\right.$ corresponds to the vacuum-sample surface). The Fourier components of the electrical field are expressed via Fourier components of the vector $\vec{D}(\vec{r})$ by using Eqs. (5) and (6). Because the lateral component of the wave vector is continuous at the interfaces, the boundary conditions (31) are valid for all Fourier components of the wave 
field. The supplementary boundary conditions follow from the continuity of the magnetic field $\vec{B} \sim \vec{\nabla} \times \vec{E}$ :

$$
\begin{aligned}
& {\left.\left[\vec{\nabla} \times \vec{D}_{\vec{K}}^{(n)}\left(1-\widetilde{\chi}_{0}^{(n)}\right)-\sum_{\vec{h}} \widetilde{\chi}_{\vec{h}} \vec{\nabla} \times \vec{D}_{(\vec{K}-\vec{h})}^{(n)}\right]\right|_{z=z_{n}}} \\
& \quad=\left.\left[\vec{\nabla} \times \vec{D}_{\vec{K}}^{(n+1)}\left(1-\widetilde{\chi}_{0}^{(n+1)}\right)-\sum_{\vec{h}} \widetilde{\chi}_{\vec{h}} \vec{\nabla} \times \vec{D}_{(\vec{K}-\vec{h})}^{(n+1)}\right]\right|_{z=z_{n}} .
\end{aligned}
$$

Equations (31) and (32) lead to the $4 N$ equations for the amplitudes $D_{\nu, s}^{(n)}$ and $D_{\nu, s}^{(n+1)}$, which deliver the wave fields in the neighbor layers. Here $n=0,1,2, \ldots, L ; L$ is a total number of layers in the sample; $n=0$ corresponds to vacuum. For convenience reasons, ${ }^{21}$ two groups of the amplitudes may be distinguished (Fig. 1). The amplitudes of the first group correspond to the wave fields decreasing inside a sample (transmitted waves with the positive imaginary part of the wave vector): $\Im u_{\nu, s}^{(n)} \equiv u_{\nu, s}^{(n)+}>0, \Rightarrow T_{\mu, s}^{(n)}, \quad \mu=1,2, \ldots, N$. The wave fields, which are increasing inside a sample, define the specular $\quad$ amplitudes: $\quad \Im u_{\nu, s}^{(n)} \equiv u_{\nu, s}^{(n)-}<0, \Rightarrow R_{\mu, s}^{(n)}, \mu$ $=1,2, \ldots, N$. Obviously, the amplitudes of the diffracted waves $T_{\mu, s, h, g_{,} . .}^{(n)}, R_{\mu, s, h, g, \ldots}^{(n)}$ are expressed through the amplitudes $T_{\mu, s}^{(n)}, R_{\mu, s}^{(n)}$ by using Eq. (30).

Thus, the total number of the amplitudes to be found, including the amplitudes in vacuum, is $4 \times N \times(L+1)$. The incident amplitudes in a vacuum

$$
T_{1, s}^{(0)}=A_{s}, \quad\left|A_{1}\right|^{2}+\left|A_{2}\right|^{2}=1,
$$

are supposed to be known. The absence of the incident waves with the wave vectors $(\vec{K}+\vec{h}),(\vec{K}+\vec{g}), \ldots$ in a vacuum gives another $2(N-1)$ equations: $\Sigma_{\mu} T_{\mu, s, h, g, \ldots}^{(0)}=0$.

Due to continuity of the lateral component of the wave vector at interfaces, the conditions (31) and (32) have to be satisfied for both principle wave and all $(N-1)$ diffracted waves taking a part in a many-beam diffraction. Hence, the boundary conditions for the wave fields at $L$ interfaces result in $4 \times N \times L$ equations. Moreover, for a typical HRXRD calculation of $\mathrm{x}$-ray scattering from thin film structures, the last layer is assumed to be infinite along the $z$ axis (substrate), and thus the reflected waves are absent: $\Sigma_{\mu} R_{\mu, s}^{(L)}$ $=0 ; \quad \Sigma_{\mu} R_{\mu, s, h, g, \ldots}^{(L)}=0$. That gives additionally $2 N$ equations. Thus, the system of linear equations is complete, and the solution delivers the observed quantity in vacuum amplitudes $R_{\mu, s}^{(0)}$ expressed via the amplitudes of the incident wave $A_{s}$.

Let us consider first the case of a single interface, i.e., LRS for the Bragg diffraction in a single crystal, and neglect the terms of order $\left|\chi_{0}\right|^{2}$ in order to compare our results with the solution of this problem in Refs. 5-7. In this case the corrections to the boundary conditions (31) due to longitudinal components of the electrical fields in the crystal are of the same order $\sim\left|\chi_{h}\right|^{2}$ and can be neglected. ${ }^{9}$ The boundary conditions (31) are then expressed independently for each polarization and are reduced to the continuity conditions for the direct and diffracted wave fields and their derivatives. With the same accuracy for the dispersion equation (24), the terms $\sim Q_{s}^{g}, \ldots$ can be omitted in the calculation of the roots near the Bragg peak for the vector $\vec{h}$. Thus, the problem is reduced to the approximate calculation of the amplitudes of the reflected waves in vacuum $\widetilde{R}_{s, h}^{(0)}, \widetilde{R}_{s, g}^{(0)}, \ldots$, independently for each reciprocal lattice vector $\vec{h}, \vec{g}, \ldots$ and using standard two-beam diffraction formulas (see, for example, Ref. 21). Thus, the observed quantity in the LRS x-ray intensity is written for the arbitrary incidence angle $\omega_{i}$ as

$$
I\left(\omega_{i}\right) \approx \frac{\cos \omega_{e}}{\cos \omega_{i}} \sum_{s=1,2}\left|\vec{e}_{s}^{h} \widetilde{R}_{s, h}^{(0)}+\vec{e}_{s}^{g} \widetilde{R}_{s, g}^{(0)}+\cdots\right|^{2} .
$$

The formula (34) coincides with the results obtained in Refs. 5-7. When summing over the reciprocal lattice vectors in Eq. (34), only the values $\vec{h}, \vec{g}, \ldots$ are taken into account, which lie along the scanning direction in the space of the momentum transfer $\vec{Q}=\vec{K}^{\prime}-\vec{K}$.

In order to estimate the contribution from many-wave diffraction, which improves the accuracy of Eq. (34), let us represent the MBD amplitudes in the form $R_{s, h}^{(0)}=\widetilde{R}_{s, h}^{(0)}$ $+r_{s, h}^{(0)}, \quad R_{s, g}^{(0)}=\widetilde{R}_{s, g}^{(0)}+r_{s, g}^{(0)}, \ldots$. The corrections $r_{s, h}^{(0)} \sim\left|\chi_{h}\right|$ should be calculated from the modified dispersion equation (24) and with the general boundary conditions (31), which mix $\sigma$ and $\pi$ polarizations. In the angular region far from the Bragg peaks $\theta_{B}^{h}<\omega_{i}<\theta_{B}^{g}$, both $\widetilde{R}_{s, h}^{(0)} \sim \widetilde{R}_{s, g}^{(0)} \sim\left|\chi_{h}\right|$ are small, therefore the contribution of the many-wave correction $\sim\left|r_{s, h}^{(0)}\right|^{2}$ into intensity is of the same order as $\widetilde{R}_{s, h}^{(0)} \times \widetilde{R}_{s, g}^{(0)}$. This means that the simulated intensity is not calculated as just a sum of the wave fields calculated in the two-beam approximation for the peaks located on the scanning direction.

Kaganer et al. ${ }^{7,17}$ demonstrated that the coherent scattering of $\mathrm{x}$ rays from the atomic planes, forming a real surface of the crystal, results in the corrections $\sim\left|\chi_{h}\right|^{2}$ for the reflected amplitudes, which are calculated for the ideal interfaces modeled by mathematical planes. This contribution into the LRS profile is additive with respect to the MBD corrections, and it can be calculated using distorted wave Born approximation, where the wave fields for ideal interfaces are used as a zeroth approximation.

To find a general solution of the boundary problem for LRS from the multilayered samples within the framework of MBD, the matrix approach ${ }^{21}$ for the two-beam diffraction case has to be extended. For simplicity reasons, we consider here the diffraction from the interface vacuum surface, which corresponds to $z=z_{0}$. The wave field is described by the set of $4 N$ plane waves ( $N$ is a number of waves in MBD). The amplitudes can be conveniently described by the $4 N$-component vector of state $|\Psi\rangle$, which has the following components in vacuum (index 0 ):

$$
\left|\Psi_{0}\right\rangle=\left(A_{1} ; A_{2} ; R_{1,0}^{(0)} ; R_{2,0}^{(0)} ; 0 ; 0 ; R_{1, h}^{(0)} ; R_{2, h}^{(0)} ; 0 ; 0 ; R_{1, g}^{(0)} ; R_{2, g}^{(0)} ; \ldots\right),
$$

where only $2 N+2$ components are nonzero. Here we account for both the nonzero amplitudes of the specularly reflected waves in vacuum $R_{s, 0}^{(0)}$ and the amplitudes of the diffracted waves $R_{s, h}^{(0)}, R_{s, g}^{(0)}, \ldots$. In the formulas, the first lower index corresponds to polarization and the second one to the reciprocal lattice vector. In the case of coplanar diffraction, when the polarizations $\vec{e}_{1,2}$ coincide with the usual $\sigma, \pi$ polariza- 
tions, the state vector in a zeroth approximation is reduced to the product of two four-component vectors as follows:

$$
\left|\Psi_{0, h}^{(0)}\right\rangle=\left|\psi_{0, h}^{(\sigma)}\right\rangle\left|\psi_{0, h}^{(\pi)}\right\rangle, \quad\left|\psi_{0, h}^{(s)}\right\rangle=\left(A_{s} ; R_{s, 0}^{(0)} ; 0 ; R_{s, h}^{(0)} ; 0 ; 0 ; \ldots\right) .
$$

The corresponding state vectors of the field in the crystal have the following components:

$$
\left|\Psi_{1}\right\rangle=\left(T_{1,1}^{(1)} ; T_{2,1}^{(1)} ; R_{1,1}^{(1)} ; R_{2,2}^{(1)} ; T_{1,2}^{(1)} ; T_{2,2}^{(1)} ; R_{1,2}^{(1)} ; R_{2,2}^{(1)} ; \ldots\right) .
$$

In the boundary conditions at the interface with coordinate $z_{i}$, the components of the state vector are multiplied by the phase factor, which depends on the corresponding root of the dispersion equation (24). In two-beam approximation, both polarizations are considered independently with accuracy $\sim\left|\chi_{\hat{h}}\right|$, and the appearance of the phase factor is a result of the multiplication of the diagonal matrix by $|\Psi\rangle .{ }^{21}$ In the MBD case, however, the mixing of the polarizations is caused by the fact that the diffraction planes of different reciprocal lattice vectors do not coincide with the incidence plane. Therefore, the polarization mixture has the same order effect as other many-wave phenomena. Using Eq. (26), the matrix $(2 N \times 2 N)$ for MBD is written in the following form:

$$
\begin{gathered}
f_{i}\left|\Psi_{i}\right\rangle \equiv\left(f_{i}\right)_{\mu, \nu, s, s^{\prime}}\left(\left|\Psi_{i}\right\rangle\right)_{\nu, s^{\prime}}, \quad \mu, \nu=1,2, \ldots 2 N, \quad s, s^{\prime}=1,2 \\
\left(f_{i}\right)_{s, s^{\prime}, \mu, \nu}=\delta_{\mu, \nu}\left(f_{\mu}^{i}\right)_{s, s^{\prime}}\left(u_{\mu}\right), \\
\left(f_{\mu}^{i}\right)_{1,1}=\left[\Phi_{1}^{2} e^{i k_{0} z_{i} u_{\mu, \sigma}}+\Phi_{2}^{2} e^{\left.i k_{0} z_{i} u_{\mu, \pi}\right],}\right. \\
\left(f_{\mu}^{i}\right)_{2,2}=\left[\Phi_{2}^{2} e^{i k_{0} z_{i} u_{\mu, \sigma}}+\Phi_{1}^{2} e^{\left.i k_{0} z_{i} u_{\mu, \pi}\right]},\right. \\
\left(f_{\mu}^{i}\right)_{1,2}=\left(f_{\mu}^{i}\right)_{2,1}=\Phi_{1} \Phi_{2}\left[-e^{i k_{0} z_{i} u_{\mu, \sigma}}+e^{\left.i k_{0} z_{i} u_{\mu, \pi}\right] .}\right.
\end{gathered}
$$

Let us derive the equations following from the $4 N$ boundary conditions (31) and (32) for the MBD case. Choosing a $y$ axis perpendicularly to the incidence plane (polarization vector $\vec{e}_{1}$ ) and $z$ axis along the surface normal (polarization vector $\vec{e}_{2}$ in the incidence plane), the conditions (31) along with Eq. (5) result in the following relations between the components of the field in vacuum (0) and in the crystal (1) at the interface $z_{i}$ :

$$
\begin{gathered}
E_{y}^{(0)}\left(z_{i}\right)=E_{y}^{(1)}\left(z_{i}\right), \Rightarrow D_{\vec{K}, 1}^{(0)}=D_{\vec{K}, 1}^{(1)}-\sum_{\vec{H}} \tilde{\chi}_{\vec{H}} D_{K-H, 1}^{(1)}, \\
D_{z}^{(0)}\left(z_{i}\right)=D_{z}^{(1)}\left(z_{i}\right), \Rightarrow D_{\vec{K}, 2}^{(0)}=D_{\vec{K}, 2}^{(1)}, \\
\frac{k_{0 z}}{k_{0}} D_{y}^{(0)}\left(z_{i}\right)=-i \frac{d}{k_{0} d z} D_{y}^{(1)}\left(z_{i}\right), \Rightarrow \frac{K_{0 z}}{k_{0}} D_{\vec{K}, 1}^{(0)}=-i \frac{d}{k_{0} d z} D_{\vec{K}, 1}^{(1)}, \\
\frac{k_{0 z}}{k_{0}} E_{z}^{(0)}\left(z_{i}\right)=-i \frac{d}{k_{0} d z} E_{z}^{(1)}\left(z_{i}\right), \Rightarrow \frac{K_{z}^{(0)}}{k_{0}} D_{\overrightarrow{K, 2}}^{(0)} \\
=-i \frac{d}{k_{0} d z}\left[D_{\vec{K}, 2}^{(1)}-\sum_{\vec{H}} \tilde{\chi}_{\vec{H}} D_{\vec{K}-\vec{H}, 2}^{(1)}\right] .
\end{gathered}
$$

Because for the many-beam diffraction the polarizations $\vec{e}_{1,2}$ do not coincide with the polarizations $\vec{e}_{\sigma, \pi}$, the derivatives in Eqs. (39) are not produced as a simple multiplication by the component $K_{z}$ of the wave vector in the sample, as it happens in two-wave diffraction. A supplementary $(2 N \times 2 N)$ matrix has to be introduced in the second and in the fourth Eqs. (39), using Eq. (38) as follows:

$$
\begin{aligned}
& f_{i}^{\prime}\left(z_{i}\right)\left|\Psi_{i}\right\rangle \equiv\left(f_{i}^{\prime}\right)_{\mu, \nu, s, s^{\prime}}\left(\left|\Psi_{i}\right\rangle\right)_{\nu, s^{\prime}}, \\
& \mu, \nu=1,2, \ldots 2 N, \quad s^{\prime}=1,2, \\
& \left(f_{i}^{\prime}\right)_{s, s^{\prime}, \mu, \nu}=\delta_{\mu, \nu}\left(\widetilde{f_{\mu}^{i}}\right)_{s, s^{\prime}}\left(u_{\mu}\right), \\
& \left(\tilde{f}_{\mu}^{i}\right)_{1,1}=\left[u_{\mu, \sigma} \Phi_{1}^{2} e^{i k_{0} z_{i} u_{\mu, \sigma}}+u_{\mu, \pi} \Phi_{2}^{2} e^{i k_{0} z_{i} u_{\mu, \pi}}\right], \\
& \left(\widetilde{f_{\mu}^{i}}\right)_{2,2}=\left[u_{\mu, \sigma} \Phi_{2}^{2} e^{i k_{0} z_{i} u_{\mu, \sigma}}+u_{\mu, \pi} \Phi_{1}^{2} e^{i k_{0} z_{i} u_{\mu, \pi}}\right], \\
& \left(\widetilde{f}_{\mu}^{i}\right)_{1,2}=\left(\widetilde{f}_{\mu}^{i}\right)_{2,1}=\Phi_{1} \Phi_{2}\left[-u_{\mu, \sigma} e^{i k_{0} z_{i} u_{\mu, \sigma}+u_{\mu, \pi}} e^{i k_{0} z_{i} u_{\mu, \pi}}\right] .
\end{aligned}
$$

For both wave field components in a vacuum and a crystal, these equations have to be resolved independently for both polarizations and for all $N$ coefficients of wave field expansion over the reciprocal lattice vectors. This requirement follows from the conservation of the wave vector components, which are parallel to the interface. As a result, the following system of $(4 N)$ linear equations is obtained for unknown components of the state field $\left|\Psi_{0}\right\rangle,\left|\Psi_{1}\right\rangle$ (we assume the surface of the sample at $z_{i}=0$ ):

$$
\begin{gathered}
A_{1}+R_{1}^{(0)}=\sum_{\mu=1}^{N}\left[T_{1 \mu}^{(1)}-\sum_{\vec{H}} \tilde{\chi}_{\vec{H}} T_{1 \mu,(\vec{K}-\vec{H})}^{(1)}\right] \\
+\sum_{\mu=1}^{N}\left[R_{1 \mu}^{(1)}-\sum_{\vec{H}} \widetilde{\chi}_{\vec{H}} R_{1 \mu,(\vec{K}-\vec{H})}^{(1)}\right], \\
\gamma_{0}\left(A_{1}-R_{1}^{(0)}\right)=\sum_{\mu=1}^{N} u_{1 \mu}^{(1)+} T_{1 \mu}^{(1)}+\sum_{\mu=1}^{N} u_{1 \mu}^{(1)-} R_{1 \mu}^{(1)}, \\
A_{2}+R_{2}^{(0)}=\sum_{\mu=1}^{N} T_{2 \mu}^{(1)}+\sum_{\mu=1}^{N} R_{2 \mu}^{(1)}, \\
\gamma_{0}\left(A_{2}-R_{1}^{(0)}\right)=\sum_{\mu=1}^{N} u_{1 \mu}^{(1)+}\left[T_{2 \mu}^{(1)}-\sum_{\vec{H}} \tilde{\chi}_{\vec{H}} T_{2 \mu,(\vec{K}-\vec{H})}^{(1)}\right] \\
+\sum_{\mu=1}^{N} u_{1 \mu}^{(1)-}\left[R_{2 \mu}^{(1)}-\sum_{\vec{H}} \tilde{\chi}_{\vec{H}} R_{2 \mu,(\vec{K}-\vec{H})}^{(1)}\right], \\
R_{1, h}^{(0)}=\sum_{\mu=1}^{N}\left[T_{1 \mu, h}^{(1)}-\sum_{\vec{H}} \widetilde{\chi}_{\vec{H}} T_{1 \mu,(\vec{K}+\vec{h}-\vec{H})}^{(1)}\right] \\
+\sum_{\mu=1}^{N}\left[R_{1 \mu, h}^{(1)}-\sum_{\vec{H}} \widetilde{\chi}_{\vec{H}} R_{1 \mu,(\vec{K}+\vec{h}-\vec{H})}^{(1)}\right],
\end{gathered}
$$




$$
-\gamma_{h} R_{1, h}^{(0)}=\sum_{\mu=1}^{N}\left(u_{1 \mu}^{(1)+}+\psi_{h}\right) T_{1 \mu, h}^{(1)}+\sum_{\mu=1}^{N}\left(u_{1 \mu}^{(1)-}+\psi_{h}\right) R_{1 \mu, h}^{(1)} .
$$

This system of equations has to imply the relations (30) between the amplitudes $T_{1 \mu, h}^{(1)}, R_{1 \mu, h}^{(1)}$ of the diffracted waves and the amplitudes $T_{1 \mu}^{(1)}, R_{1 \mu}^{(1)}$ of the direct waves. Contrary to the two-beam diffraction, both polarizations are bounded because of the nondiagonal matrices $V_{s, s^{\prime}, \mu}^{h}$ in Eq. (30).

In the general case of $L$ layers, $(L+1)$ state vectors of the electromagnetic field have to be found, starting from the vacuum (35) and proceeding with the successive $L$ vectors as follows:

$$
\begin{aligned}
\left|\Psi_{n}\right\rangle= & \left(T_{1,1}^{(n)} ; T_{2,1}^{(n)} ; R_{1,1}^{(n)} ; R_{2,1}^{(n)} ; T_{1,2}^{(n)} ; T_{2,2}^{(n)} ; R_{1,2}^{(n)} ; R_{1,2}^{(n)}\right. \\
& \left.\ldots T_{1, N}^{(n)} ; T_{2, N}^{(n)} ; R_{1, N}^{(n)} ; R_{2, N}^{(n)}\right) \\
& n=1,2 \ldots(L-1)
\end{aligned}
$$

$$
\left|\Psi_{L}\right\rangle=\left(T_{1,1}^{(L)} ; T_{2,1}^{(L)} ; 0 ; 0 ; T_{1,2}^{(L)} ; T_{2,2}^{(L)} ; 0 ; 0 ; \ldots T_{1, N}^{(L)} ; T_{2, N}^{(L)} ; 0 ; 0\right) .
$$

Thus, the MBD boundary conditions at $L$ interfaces, with the coordinates $z_{0}=0$ on the surface and $z_{n}, n=1,2, \ldots(L-1)$ between other layers, are represented by the matrix equations

$$
\begin{gathered}
\hat{S}_{0}\left(z_{0}\right)\left|\Psi_{0}\right\rangle=\hat{S}_{1}\left(z_{0}\right)\left|\Psi_{1}\right\rangle, \\
\hat{S}_{1}\left(z_{1}\right)\left|\Psi_{1}\right\rangle=\hat{S}_{2}\left(z_{1}\right)\left|\Psi_{2}\right\rangle, \\
\cdots \\
\hat{S}_{L-1}\left(z_{L-1}\right)\left|\Psi_{L-1}\right\rangle=\hat{S}_{L}\left(z_{L}\right)\left|\Psi_{L}\right\rangle,
\end{gathered}
$$

where the expressions for $4 N \times 4 N$ matrices $\hat{S}$ follow from Eqs. (38), (39), and (41), and the expression for the $4 N$ $\times 4 N$ matrix can be built as follows:

$$
\begin{gathered}
s_{s p,-, j}=\vec{e}_{(3-j)} \cdot\left(-k_{0} \gamma_{0} \vec{Z}+\vec{k}_{\perp}\right) \times \vec{e}_{\pi}^{(s p)} \frac{1}{k_{0}}, \gamma_{2 \pi}=-\vec{e}_{2} \cdot \vec{e}_{\pi}^{(s p)}, \\
\gamma_{1 \pi}=-\vec{e}_{1} \cdot \vec{e}_{\pi}^{(s p)}, \quad s_{l}^{k,+, j}=\delta_{k, l} \vec{e}_{j} \cdot \vec{e}_{s}^{(k)}, \\
s_{l}^{k,-, j}=\delta_{k, l} \vec{e}_{(3-j)} \cdot\left(-k_{0} \gamma_{h_{k}}+\vec{k}_{\perp}+\vec{h}_{k_{\perp}}\right) \times \vec{e}_{s}^{(k)} \frac{1}{k_{0}} \\
l=\{1, \ldots, N ; s=(\sigma, \pi)\}, \\
S_{n}=\left(\begin{array}{ccc}
s_{(\mu=1,+, \sigma)}^{(\nu=1,+, 1)} & \cdots & s_{(\mu=N,-, \pi)}^{(\nu=1,+1)} \\
\vdots & \ddots & \vdots \\
s_{(\mu=1,+,+\sigma)}^{(\nu=N,-2)} & \cdots & s_{(\mu=N,-, \pi)}^{(\nu=N,-, 2)}
\end{array}\right)
\end{gathered}
$$

$$
\begin{aligned}
& s_{\mu}^{1,+, j}= e^{i k_{0} u_{\mu} z^{(n)}} \vec{e}_{j} \cdot\left\{\vec{e}_{\mu}\left(1-\tilde{\chi}_{0}\right)+\tilde{\chi}_{0} \vec{Z}\left(\vec{Z} \cdot \vec{e}_{\mu}\right)\right. \\
&\left.-\sum_{\vec{h}} \widetilde{\chi}_{h}\left[\vec{V}_{\mu}^{h}-\vec{Z}\left(\vec{z} \cdot \vec{V}_{\mu}^{h}\right)\right]\right\}, \\
& s_{\mu}^{1,-, j}=e^{i k_{0} u_{\mu} z^{(n)}} \vec{e}_{(3-j)} \cdot\left(k_{0} u_{\mu} \vec{Z}+\vec{k}_{\perp}\right) \\
& \\
& \quad \times\left(\vec{e}_{\mu}\left(1-\tilde{\chi}_{0}\right)-\sum_{\vec{h}} \tilde{\chi}_{\vec{h}} \vec{V}_{\mu}^{\vec{h}}\right) \frac{1}{k_{0}},
\end{aligned}
$$

$\mu=\{1, \ldots, N, \pm, s(=\sigma, \pi)\}, \quad \nu=\{1, \ldots, N, \pm, j=1,2\}$, 


$$
\begin{aligned}
s_{\mu}^{m,-, j}= & e^{i k_{0} u_{\mu} z^{(n)}} \vec{\chi}_{h_{m}} \vec{e}_{(3-j)} \cdot\left(k_{0} u_{\mu} \vec{Z}+\vec{k}_{\perp}+\vec{h}_{m}\right) \\
& \times\left[\vec{V}_{\mu}^{h_{m}}\left(1-\tilde{\chi}_{0}\right)-\vec{e}_{\mu}\right] \frac{1}{k_{0}} .
\end{aligned}
$$

The wave field in the vacuum can be written in the following form:

$$
\begin{aligned}
\vec{E}(\vec{r})= & \sum_{s=\sigma, \pi} e^{i \vec{k}_{\perp} \cdot \vec{r}_{\perp}}\left(A_{s}^{(0)} e^{i k_{0} \gamma_{0} z} \vec{e}_{s}^{(0)}+A_{s}^{(s p)} e^{-i k_{0} \gamma_{0} z} \vec{e}_{s}^{(s p)}\right. \\
& \left.+\sum_{j=1}^{N-1} R_{j, s} e^{i \vec{h}_{j_{\perp}} \cdot \vec{r}_{\perp}} e^{-i k_{0} \gamma_{h_{j}} z} e_{s}^{(j)}\right)
\end{aligned}
$$

where $A_{s}^{(s p)} \equiv R_{s, 0}^{(0)}$ are the amplitudes of the specular reflected waves and the polarization vectors are represented analogously to Eq. (10) as follows:

$$
\begin{gathered}
\vec{e}_{\sigma}^{(0)}=\vec{e}_{1}, \quad \vec{e}_{\pi}^{(0)}=\vec{e}_{2}, \quad e_{\sigma}^{(s p)}=\vec{e}_{1}, \\
e_{\pi}^{(s p)}=-\left(-k_{0} \gamma_{0} \vec{Z}+\vec{k}_{\perp}\right) \times \vec{e}_{\sigma}^{(s p)} \frac{1}{k_{0}}, \\
e_{\sigma}^{(j)}=\left(-k_{0} \gamma_{h_{j}}+\vec{k}_{\perp}+\vec{h}_{j_{\perp}}\right) \times \vec{Z}, \\
e_{\pi}^{(j)}=-\left(-k_{0} \gamma_{h_{j}}+\vec{k}_{\perp}+\vec{h}_{j_{\perp}}\right) \times e_{\sigma}^{(j)} \frac{1}{k_{0}} .
\end{gathered}
$$

The further calculations are formally similar to the two-beam diffraction case. The matrix $\hat{M}$ connecting the amplitudes in a vacuum and substrate is found by the solution of the equation system (43) as follows:

$$
\begin{aligned}
\left|\Psi_{0}\right\rangle= & \hat{M}\left|\Psi_{L}\right\rangle, \\
& \hat{M}=\hat{S}_{0}^{-1} \hat{S}_{1}\left(z_{0}\right)\left[\hat{S}_{1}\left(z_{1}\right)\right]^{-1} \cdots\left[\hat{S}_{L-1}\left(z_{L}\right)\right]^{-1} \hat{S}_{L}\left(z_{L}\right) .
\end{aligned}
$$

As mentioned above, for each vector of the wave field at the interface, the vector components can be separated into the amplitudes of the transmitted and the reflected waves as follows:

$$
\left|\Psi_{0}\right\rangle=\left|\left\{T_{0}\right\} ;\left\{R_{0}\right\}\right\rangle ; \quad\left|\Psi_{L}\right\rangle=\left|\left\{T_{L}\right\} ;\{0\}\right\rangle,
$$

where the components $\left\{T_{0}\right\}$ possess only two nonzero amplitudes $A_{1}, A_{2}$. The matrix can also be split into blocks of $(2 N \times 2 N)$ matrices as follows:

$$
\hat{M}=\left(\begin{array}{cc}
\hat{M}^{T T} & \hat{M}^{T R} \\
\hat{M}^{R T} & \hat{M}^{R R}
\end{array}\right) .
$$

Then, the matrix solution of Eq. (51) for unknown amplitudes of the reflected waves is

$$
\left|\left\{R_{0}\right\}\right\rangle=\hat{M}^{R T}\left[\hat{M}^{T T}\right]^{-1}\left|\left\{T_{0}\right\}\right\rangle .
$$

Finally, the intensity of $\mathrm{x}$-ray radiation in the LRS is calculated as

$$
I\left(\omega_{i}\right)=\frac{\cos \omega_{e}}{\cos \omega_{i}}\left|\sum_{s=1,2} \sum_{j=1}^{N} R_{s, j} \vec{e}_{s}^{(j)}\right|^{2},
$$

with $R_{s, h} ; R_{s, g} ; \ldots$, defined by the components of the vector $\left|\left\{R_{0}\right\}\right\rangle$.

\section{DISCUSSION AND COMPARISON WITH THE EXPERIMENTAL DATA}

The typical samples measured in a HRXRD setup consist of thin films on a substrate. Both substrate and film have monocrystalline structure and the thickness of the film is comparable with the x-ray extinction length. Therefore, the dynamical theory has to be used to calculate the position, the shape, and the intensity of the Bragg peaks from the substrate and layers in the vicinity of Bragg reflections. Usually, within the two-beam approximation for the dynamical diffraction theory, the peaks for each vector $\vec{h}$ are simulated by using the deviation parameter $\alpha_{h}=\left(2 \vec{k} \vec{h}+h^{2}\right) / k_{0}^{2}$ from the exact Bragg condition and for the polarization vectors corresponding to the definite reciprocal lattice vector $\vec{h}$. This deviation parameter, however, does no longer yield a valid result in the LRS calculations near reflections other than $\vec{h}$ reflection $[\vec{g}, \vec{f}, \ldots$ (see Fig. 1$)]$, where other deviation parameters and polarization vectors have to be introduced. However, the use of different deviation parameters for a single scan calculation complicates essentially the fitting of the experimental LRS data.

The method reported in this work, being valid for the entire scanning angular range, solves the problem of the covariant simulation of the LRS diffraction curve from multilayered samples. The $\mathrm{x}$-ray diffraction profile has a covariant form depending on the incidence angle of $\mathrm{x}$ rays and is not related to the particular reciprocal lattice vector. The problem is solved by using the analytical expressions (27) and (28) for the roots of the MBD dispersion equation and the generalized matrix method for the boundary conditions in multilayered samples (43).

The theory presented in this work also gives the answer to the principal question formulated in Ref. 4: how important is the role of many-wave effects and the corrections $\sim|\chi|^{2}$ for the calculation of LRS between the Bragg peaks? The accuracy of the HRXRD profiles measured with modern instruments achieves the value of $\xi_{\text {exp }} \sim 10^{-7}, 3$ and the magnitude of the parameter describing the ratio of $\mathrm{X}$-ray intensities on the wings $\left(I_{W}\right)$ and on the maximum $\left(I_{B}\right)$ of the Bragg peak is

$$
\xi_{W / B}=\frac{I_{W}}{I_{B}} \sim\left|\chi_{h}\right| \sim 10^{-4}-10^{-5}>\xi_{\text {exp }} .
$$

Thus, the intensity oscillations are quite observable in the high-resolution setup. The condition at which DDT has to replace the kinematical theory for correct data interpretation also includes the ratio of the film thickness $L$ and the extinction length $L_{\text {ext }}$ as follows: 


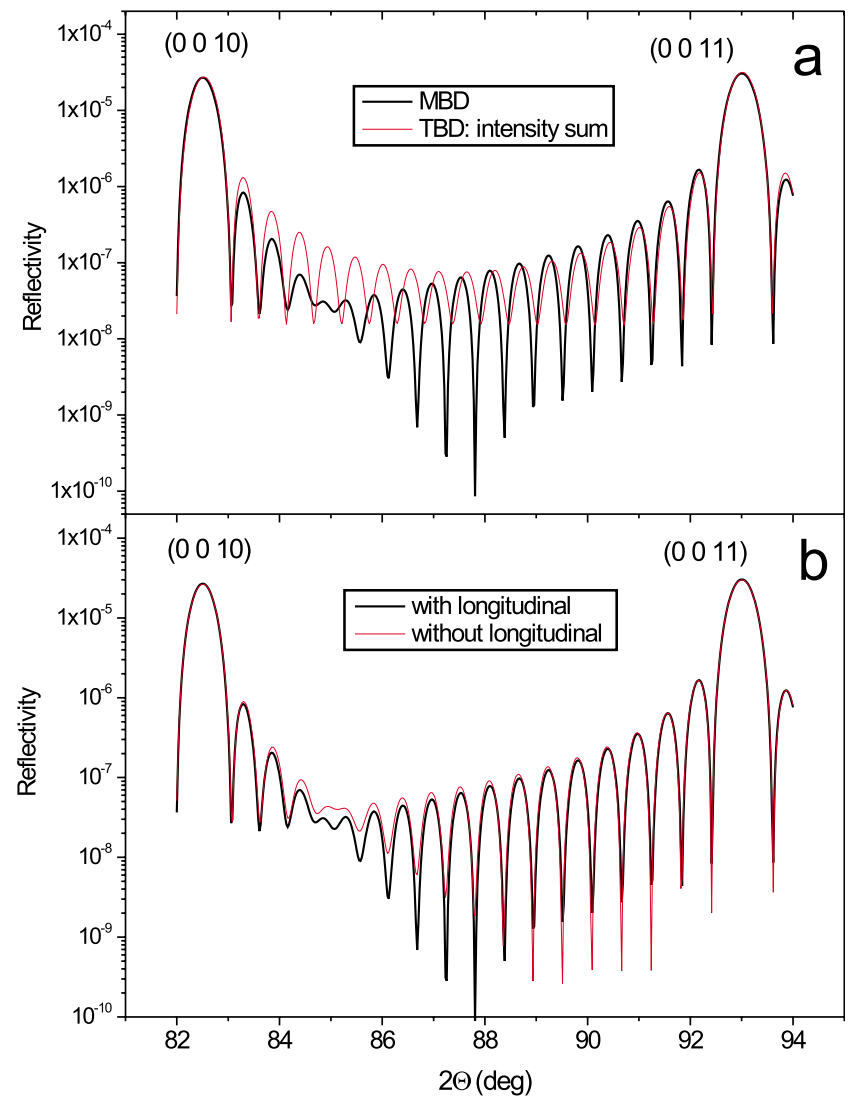

FIG. 2. (Color online) (a) Diffracted x-ray intensity between Bragg peaks, calculated by the MBD (thick black line) and twobeam diffraction method using intensity summation (thin red line). (b) MBD calculations with (thick black line) and without (thin red line) accounting for the longitudinal component of the wave field in the boundary conditions.

$$
\xi_{D / K}=k_{0} L\left|\chi_{0}\right| \equiv \frac{L}{L_{e x t}} \sim 1 .
$$

As follows from the dispersion equation (27), the calculation of x-ray intensity for a stand-alone Bragg peak within the two-beam diffraction approach means neglecting the contributions from the neighboring waves, which are determined by the value $\xi_{2}=\left|\chi_{h}\right|^{2}$. This value also describes the changes in the boundary conditions (31) comparing to the two-beam case due to the transverse component of the wave fields (polarization effects). The phase shift of the waves in media is then quantified by the parameter

$$
\xi_{M B D}=k_{0} L\left|\chi_{h}\right|^{2} \sim k_{0} L_{e x t}\left|\chi_{h}\right|^{2} \sim\left|\chi_{h}\right|,
$$

and thus $\xi_{M B D} \sim \xi_{W / B}$. Therefore, the many-wave and polarization effects may show up in the regions between the peaks.

The effectiveness of the algorithm proposed in this work is proven for a measured LRS diffraction profile from a 22 nm layer of $\mathrm{YBa}_{2} \mathrm{Cu}_{3} \mathrm{O}_{7}$ on the $\mathrm{SrTiO}_{3}$ substrate, corresponding to the (001) truncation rod and with the reflection indices $1 \leq l_{S} \leq 4$ for the substrate and $1 \leq l_{S} \leq 13$ for the film. The thickness of the monocrystalline film $L$ is one-tenth of the extinction length $L_{e x t}$. All the measurements and simu-

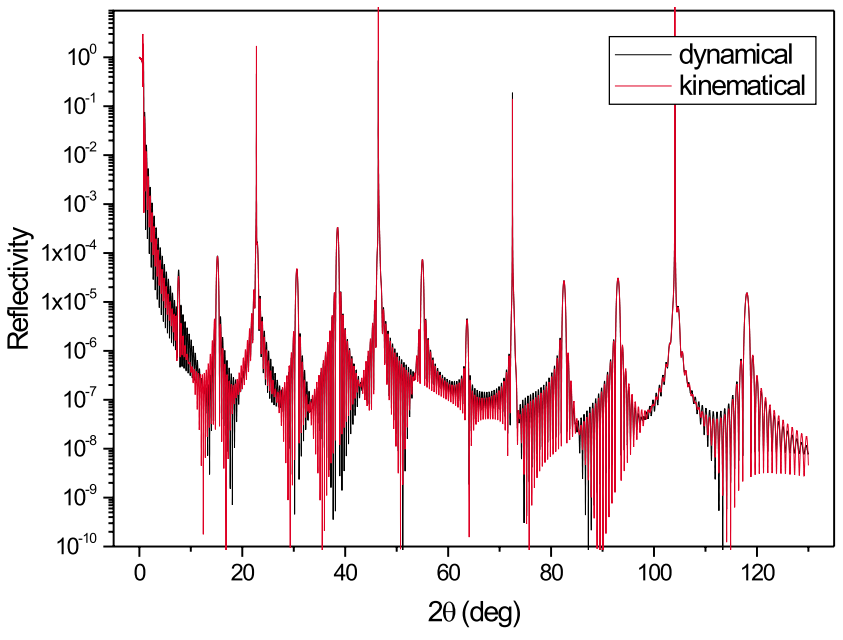

FIG. 3. (Color online) Long-range scans for the $22 \mathrm{~nm}$ $\mathrm{YBa}_{2} \mathrm{Cu}_{3} \mathrm{O}_{7}$ layer on the $\mathrm{SrTiO}_{3}$ substrate calculated by the kinematical (red line) and the dynamical (black line) theories. The kinematical profile in the specular reflection area has been calculated by Parratt's formulas.

lations are performed at the $\mathrm{Cu} K \alpha$ x-ray wavelength.

First, we demonstrate the influence of the many-beam effects on the profile shape between the Bragg peaks. Figure 2(a) shows the LRS segment between $\left(\begin{array}{lll}0 & 0 & 10\end{array}\right)$ and $\left(\begin{array}{lll}0 & 0 & 11\end{array}\right)$ peaks calculated using Eq. (54) on the basis of MBD (thick black line) and two-beam dynamical diffraction theory (thin red line) by the direct summing of x-ray intensities from both reflections, which results in the intensity's discrepancies between the peaks. However, as was shown in Refs. 5-7 that the precision of the two-beam approach may be improved when the amplitudes instead of intensities of the wave fields from different Bragg reflections are summed up. However, the LRS diffracted intensity also evidently changes between the Bragg peaks, when the MBD boundary conditions (39) take into account the longitudinal component of the electromagnetic field in the crystal. Figure 2(b) shows the same LRS segment simulated with (thick black line) and without (thin red line) a longitudinal component in the boundary conditions. The amplitude of the MBD corrections is seen to be consistent with the estimates above.

Figure 3 compares the LRS profile for the discussed sample calculated by dynamical MBD (black line) and by the kinematical theory (red line). ${ }^{16}$ The simulations confirm the general agreement of both approaches. However, the difference in both profiles is prominently evident in the regions of Bragg peak [Fig. 4(a)] and at the small incidence angle of $x$ rays [Fig. 4(b)], where specularly reflected waves are dominating. These discrepancies may be observed in highresolution experiments due to the high precision of modern diffractometers.

Finally, the proposed theory has been verified on experimental data from a $\mathrm{YBa}_{2} \mathrm{Cu}_{3} \mathrm{O}_{7} / \mathrm{SrTiO}_{3}$ sample. Figure 5 compares the measured data (red line) and simulated (black line) by the current MBD algorithm diffraction profile, which accounts for 16 scattered waves. The figure demonstrates a good agreement of the positions and the intensity ratio of the Bragg peaks between the measured and calculated LRS 

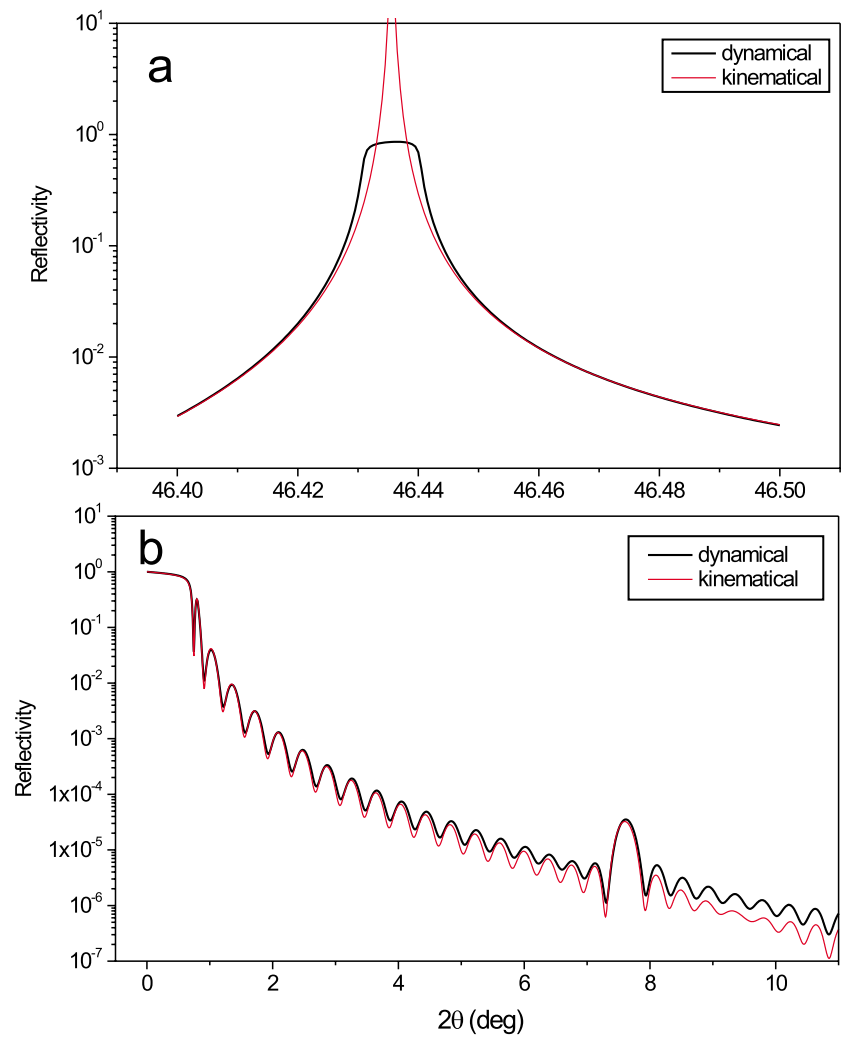

FIG. 4. (Color online) Dynamical (thick black line) vs kinematical (thin red line) simulations in the Bragg peak (a) and in specular reflection area (b).

curves. The theoretical curve is calculated also in the specular reflection region. The measured profile does not contain specular reflection because the experimental setup requires the suppression of the diffuse $\mathrm{x}$-ray scattering by a knife edge, which is not desirable in HRXRD measurements.

\section{CONCLUSIONS}

A theoretical approach based on many-beam dynamical $\mathrm{x}$-ray diffraction is developed for the calculation of the wide

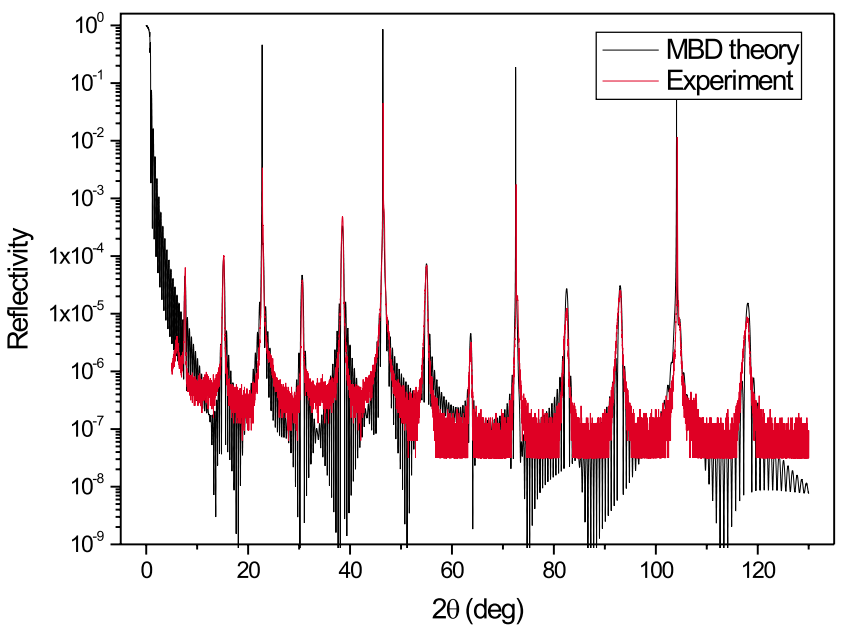

FIG. 5. (Color online) Simulated by the MBD (red line) and measured (black line) long-range scans for the $22 \mathrm{~nm} \mathrm{YBa}_{2} \mathrm{Cu}_{3} \mathrm{O}_{7}$ layer on the $\mathrm{SrTiO}_{3}$ substrate.

$\mathrm{x}$-ray diffraction profiles from multilayered crystalline samples in coplanar geometry and could be used in the noncoplanar geometry. The corrections of the second order on the $\mathrm{x}$-ray polarizability are taken into account in the dispersion equations and in the boundary conditions. The major part of the algorithm is obtained in an analytical form, from which it is possible to write a numerical code for the simulation of long-range scans for HRXRD, based upon the matrix method for $\mathrm{x}$-ray diffraction from multilayered structures. The influence of various factors upon the simulation of $\mathrm{x}$-ray profiles containing multiple Bragg reflections is investigated and the comparison with experimental data is presented.

\section{ACKNOWLEDGMENTS}

The authors thank H. Guérault and R. Eisenhower (Bruker AXS, Karlsruhe) for the measurements and discussions. A.I.B. and I.F. appreciate the support from the Ministry of Education of the Republic of Belarus in the scope of the program "Crystalline and molecular structures" (objective 03.02).
${ }^{1}$ Nondestructive Methods for Materials Characterization, edited by G. Y. Baaklini, N. Meyendorf, T. E. Matikas, and R. S. Gilmore (Materials Research Society, Pittsburgh, 2000), Vol. 591.

${ }^{2}$ D. B. Williams and C. B. Carter, Transmission Electron Microscopy (Plenum, New York, 1996).

${ }^{3}$ U. Pietsch, V. Holy, and T. Baumbach, X-ray Scattering: From Thin Films to Lateral Nanostructures (Springer Verlag, Heidelberg, 2004).

${ }^{4}$ V. Holy and P. F. Fewster, J. Appl. Crystallogr. 41, 18 (2008).

${ }^{5}$ S. G. Podorov, N. N. Faleev, K. M. Pavlov, D. M. Paganin, S. A. Stepanov, and E. Förster, J. Appl. Crystallogr. 39, 652 (2006).

${ }^{6}$ K. M. Pavlov, D. M. Paganin, D. J. Vine, and L. Kirste, Phys. Status Solidi A 204, 3613 (2007).
${ }^{7}$ V. M. Kaganer, Phys. Rev. B 75, 245425 (2007).

${ }^{8}$ I. K. Robinson, Handbook of Synchrotron Radiation (Elsevier, Amsterdam, 1991), Vol. 3, Chap. 7, p. 221; E. Vlieg, J. Appl. Crystallogr. 33, 401 (2000).

${ }^{9}$ A. Authier, Dynamical Theory of X-ray Diffraction (International Union of Crystallography, Oxford, 2003).

${ }^{10}$ W. J. Bartels, J. Hornstra, and D. J. W. Lobeek, Acta Crystallogr., Sect. A: Found Crystallogr. 42, 539 (1986).

${ }^{11}$ A. M. Afanas'ev, A. P. Aleksandrov, S. S. Fanchenko, V. A. Chaplanov, and S. S. Yakimov, Acta Crystallogr., Sect. A: Found. Crystallogr. 42, 116 (1986).

${ }^{12}$ R. Colella, Phys. Rev. B 43, 13827 (1991).

${ }^{13}$ A. Caticha, Phys. Rev. B 47, 76 (1993).

${ }^{14}$ A. Caticha, Phys. Rev. B 49, 33 (1994). 
${ }^{15}$ S. Zamir, O. Steinberg, U. Tisch, J. Salzman, and E. Zolotoyabko, J. Phys. D, 38, A239 (2005); E. Zolotoyabko, J. Appl. Crystallogr. 31, 241 (1998).

${ }^{16}$ I. K. Robinson and D. J. Tweet, Rep. Prog. Phys. 55, 599 (1992).

${ }^{17}$ V. M. Kaganer, B. Jenichen, R. Shayduk, and W. Braun, Phys. Rev. B 77, 125325 (2008).

${ }^{18}$ V. Holy and P. F. Fewster, J. Appl. Crystallogr. 41, 18 (2008).

${ }^{19}$ S. Takagi, Acta Crystallogr., 15, 1311 (1962); D. Taupin, Bull.
Soc. Fr. Mineral. Cristallogr. 87, 469 (1964).

${ }^{20}$ R. Zaus, J. Appl. Crystallogr. 26, 801 (1993).

${ }^{21}$ S. A. Stepanov, E. A. Kondrashkina, R. Köhler, D. V. Novikov, G. Materlik, and S. M. Durbin, Phys. Rev. B 57, 4829 (1998).

${ }^{22}$ X.-R. Huang and M. Dudley, Acta Crystallogr. 59, 163 (2003); W. Cho, X.-R. Huang, and M. Dudley, ibid. 60, 195 (2004).

${ }^{23}$ J. D. Jackson, Klassische Elektrodynamik (Walter de Gruyter Verlag, Berlin, 1982). 